\title{
Caracterización del registro cerámico del sitio Barranca I (Dpto. Río Cuarto, Córdoba)
}

\author{
Characterization of the ceramic record from Barranca I \\ site (Dpto. Río Cuarto, Córdoba)
}

RMA

\author{
VI Jornadas \\ Arqueológicas Cuyanas \\ Denis Ezequiel Reinoso
}

CONICET-UNRC, E-mail: denisreinoso@gmail.com

\begin{abstract}
Resumen
En el presente trabajo se exponen los resultados iniciales del análisis ceramológico del Componente 1 del sitio Barranca I, fechado en LP- 2677 - $290 \pm 50$ años AP, localizado en el área del piedemonte de la sección oriental de la Sierra de Comechingones (Dpto. Río Cuarto, Córdoba, Argentina).

Se examinan aspectos tecnológicos, morfológicos, funcionales y estilísticos de un registro caracterizado por la homogeneidad de las pastas a nivel macroscópico y un alto grado de fragmentación de los tiestos. El objetivo del análisis de estos materiales fue lograr una aproximación al comportamiento de estas sociedades en lo que respecta a pautas de manufactura y uso de los diferentes contenedores cerámicos, de acuerdo al entorno ambiental del monte del espinal.
\end{abstract}

Palabras Clave: Cerámica prehispánica; Sierra de Comechingones; Análisis Macroscópicos; Iconografía; Antiplásticos.

\begin{abstract}
This paper exposed the initial results of the ceramic analysis of the Component 1 from the site Barranca I, dated $2677 \mathrm{LP}$ - $290 \pm 50$ years BP, located in the area of the foothills of the eastern section of the Sierra de Comechingones (Dept. Río Cuarto, Córdoba, Argentina).

Technological, morphological, functional and stylistic aspects of a record characterized by the homogeneity of ceramic pastas at the macroscopic level and a high degree of fragmentation of the pots are considered here. The objective of the analysis of these materials was obtain an approximate the behavior of these societies with respect to patterns of manufacture and use of the various ceramic containers, according to the ambient environment of espinal.
\end{abstract}

Keywords: Pre-hispanic ceramics; Sierra de Comechingones; Macroscopic Analysis; Iconography;Antiplastics.

Situado en el piedemonte oriental de la Sierra de Comechingones (Córdoba, Argentina) el sitio Barranca I ofrece valiosa información sobre el período Holocénico Tardío en la región ${ }^{1}$. En este trabajo se procura indagar sobre las características tecnológicas, formas y decoración de la cerámica -fundamentalmente a nivel macroscópicode uno de los componentes arqueológicos del depósito. Para esto se optó por seguir los procedimientos analíticos delineados por Cremonte y Bugliani (2006-2009) atendiendo a las tres dimensiones elementales de los contenedores cerámicos: pasta, forma e iconografía. Todo esto a los fines de lograr un primer acercamiento a las diversas pautas conductuales implicadas en la elaboración y uso de los distintos contenedores y elementos cerámicos.

\footnotetext{
1 Los resultados más recientes de las características del sitio se presentaron en el VI Congreso Nacional de Arqueometría realizado en la ciudad de Río Cuarto (Rocchietti y Ribero 2015b)
}

\section{Ubicación del sitio}

El sitio Barranca I se localiza, como su nombre lo indica, sobre una alta barranca sedimentaria en la margen derecha del Río Piedra Blanca, a escasos kilómetros río arriba del puente homónimo por el que cruza la ruta provincial número 23, cercano a la turística localidad de Las Albahacas, en el área serrana noroeste del departamento Río Cuarto, Córdoba (Figura 1). Se ubica en un área particular del piedemonte serrano, donde la geología parece haber orientado activamente las formas de asentamiento y movilidad de los grupos humanos, quienes habrían desplegado gran parte de sus actividades en las márgenes del río y arroyos circundantes. El Piedra Blanca discurre en esta área sobre una formación distrital de rocas metamórficas que no ofrece grandes aleros de refugio, siendo así más habitual el registro de emplazamientos al aire libre (Rocchietti y Ribero 2015a). 

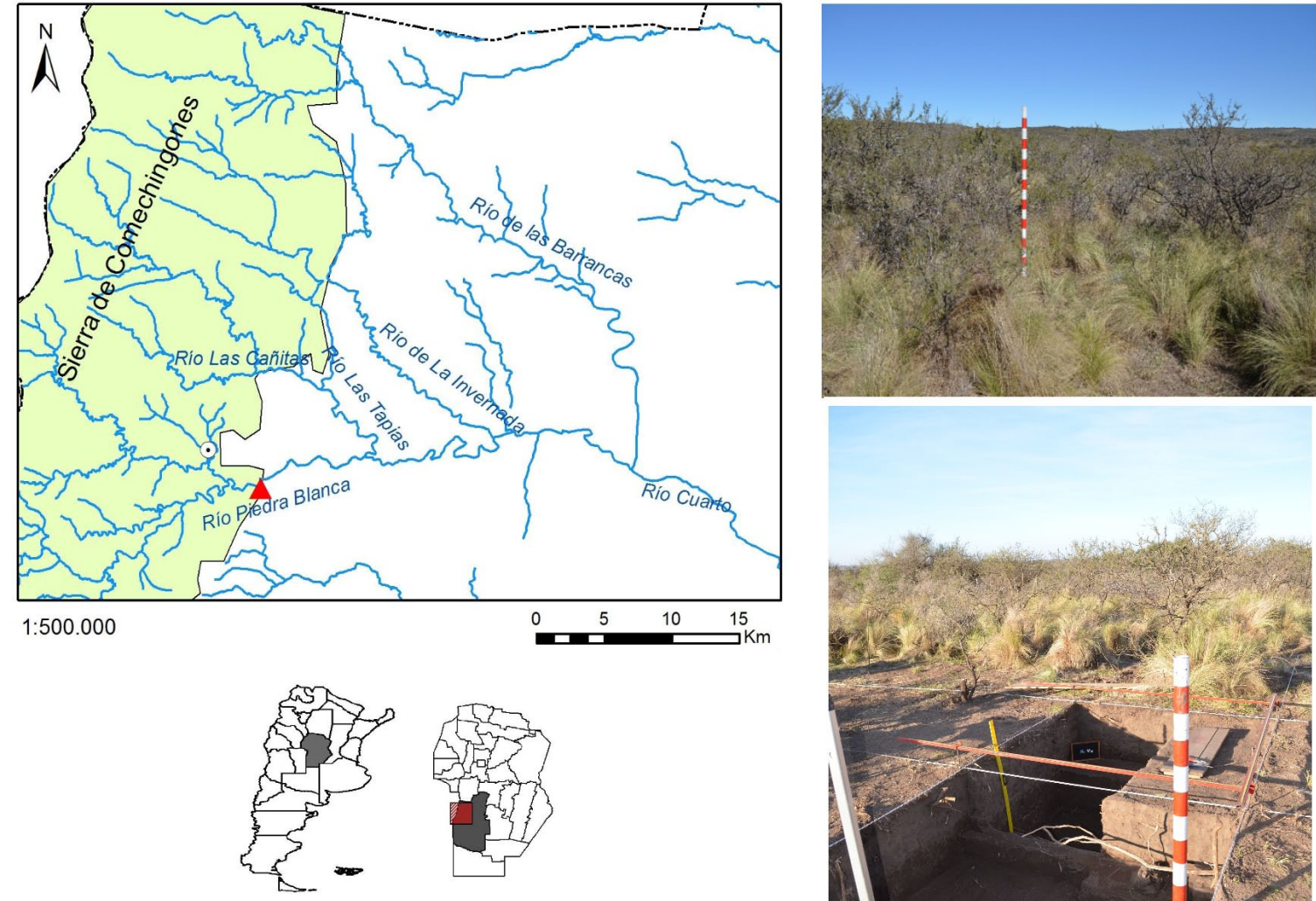

Referencias

\begin{tabular}{|c|c|c|}
\hline I_-..jLímites departamentales & $\odot$ Las Albahacas & $\Delta$ Sitio Barranca I \\
\hline$\frown$ Ríos & $\square$ Sierra de Comechingones & \\
\hline
\end{tabular}

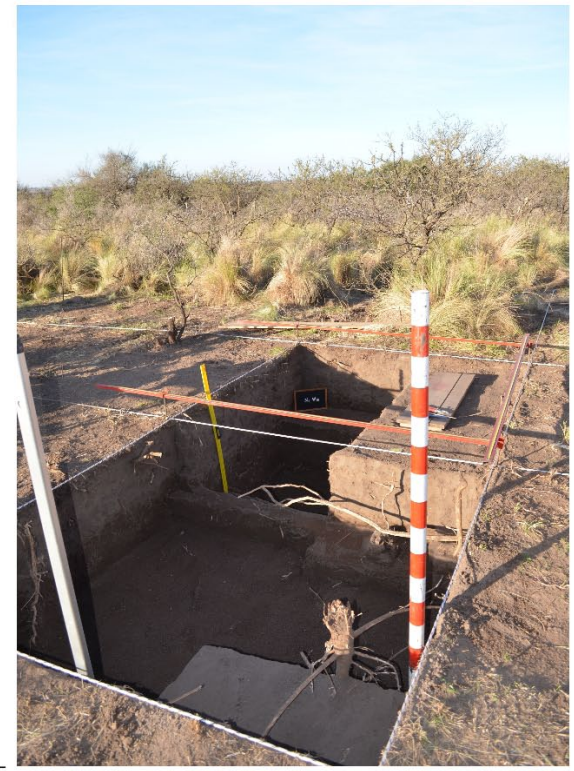

Figura 1: Ubicación y vistas del sitio Barranca I.

Figure 1: Location and views of Barranca I site.

\begin{tabular}{ccccccccc} 
& \multicolumn{2}{c}{ Fragmento de contenedor } & \multicolumn{2}{c}{ Decoración } & \multicolumn{3}{c}{ Aspectos morfológicos } \\
& Sí & No & Sí & No & Borde & Base & Cuerpo \\
\hline C1 & 508 & 5 & 28 & 480 & 32 & 9 & 467 \\
\hline
\end{tabular}

Tabla 1: Clasificación general de los materiales del Componente I, sitio Barranca I.

Table 1: Component I materials general classification, Barranca I site.

El componente tecnológico del Barranca I se correlaciona con el presente en otros sitios de la formación arqueológica regional que Austral y Rocchietti (2004) definieron como ceramolítico. La misma se caracteriza por presentarse en forma de yacimientos con abundante distribución de talla y desechos, artefactos con poca inversión de trabajo en su manufactura, mayoritariamente raspadores, puntas de proyectil apedunculadas de modulo pequeño, con ocasional presencia de elementos de molienda; la cerámica que aparece en poca cantidad, es de una elaboración expeditiva y en escasa proporción decorada; la fracción ósea es todavía mucho menor y a veces inexistente (Austral y Rocchietti 2004).
Las excavaciones realizadas en el año 2011 permitieron identificar al menos dos componentes arqueológicos en estratigrafía (Rocchietti et al. 2013), de acuerdo al estado actual de la investigación de laboratorio en el presente trabajo se desarrolla la caracterización del material cerámico del componente estratigráficamente superior, denominado Componente 1 y fechado en LP- 2677 $290 \pm 50$ años AP. El fechado resulta contemporáneo al ingreso de los conquistadores españoles en las Sierras Centrales de Córdoba (Pastor y Medina 2013), sin embargo las pautas tecnológicas presentes en los materiales recuperados corresponden a las existentes en el amplio espectro cronológico prehispánico de los sitios 
Figura 2: Elementos representativos de la muestra: "a": fragmento de estatuilla, "b": fragmento de base, "c": bordes.

Figure 2: Representative items of the sample: "a": statuette fragment, " $b$ " base fragment, " $c$ ": edges.
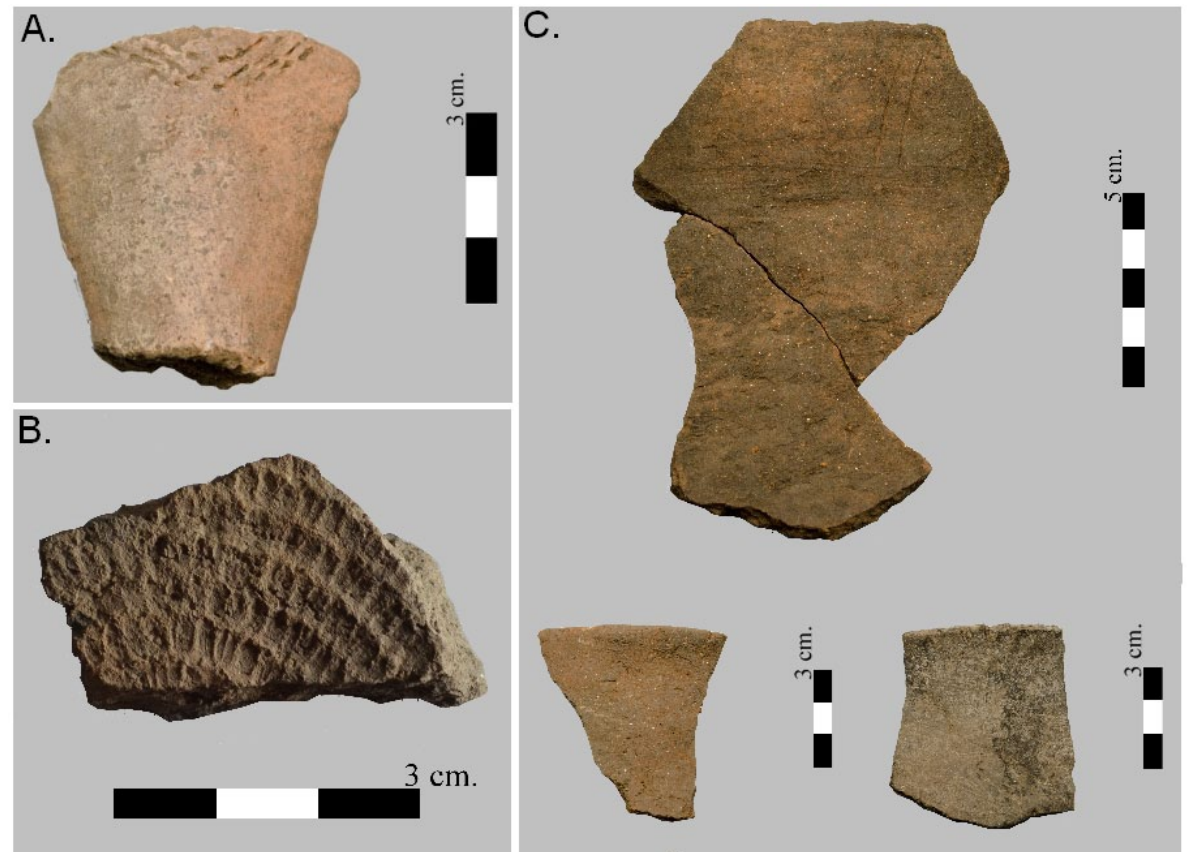

ceramolíticos de la región (Austral y Rocchietti 2004). Además, en toda la amplia área excavada no se recuperó ningún elemento de origen europeo tal como metal, vidrio o cerámica exótica. Estos elementos, aunque no permiten desestimarlo, no evidencian la presencia o influencia de europeos en los momentos de ocupación del sitio.

Es preciso mencionar que además de la cerámica el conjunto tecnológico de este componente posee desechos de talla en cantidad y artefactos de formatización somera, mayoritariamente en cuarzo, aunque con algunos registros en calcedonia y ópalo. Se hallaron puntas de proyectil apedunculadas de tamaño pequeño, raspadores de diverso tamaño y forma, percutores y elementos de molienda. El material óseo no es muy abundante y en los estudios realizados hasta el momento no se identificaron elementos formatizados.

\section{El conjunto cerámico}

El registro cerámico estudiado está compuesto por más de 500 fragmentos, de entre $0,5 \mathrm{~cm} \times 0,5 \mathrm{~cm}$ y $10 \mathrm{~cm} \times$ $9 \mathrm{~cm}$. El espesor es muy variable, pero oscila entre los 0,4 $\mathrm{cm}$ y los 1,2 cm (Tabla 1).

Además de los fragmentos de contenedores, se hallaron torteros íntegros, inconclusos y fragmentados, el torso de una estatuilla antropomorfa (Figura 2 A) que revela en su segmento transversal más ancho la presencia decorativa de líneas punteadas mediante impresión en forma de $V$ que se unen al centro de lo que sería el pecho de la figura. Los motivos y la técnica de decoración sobre la estatuilla repiten elementos presentes en otros tiestos decorados hallados, los antiplásticos serían los mismos aunque con una granulometría mayor y levemente menos homogénea en su distribución. Éste fragmento es un registro inexistente en otros sitios de la formación ceramolítica.

\section{Análisis de Pastas}

El análisis de las pastas fue realizado mediante el empleo de lupa binocular e incipientemente mediante microscopio petrográfico, buscando identificar y caracterizar atributos generales de las materias primas empleadas, como su estructura, abundancia y distribución. Se siguieron los criterios de clasificación propuestos por Cremonte y Bugliani (2006-2009).

Para todos los fragmentos de la muestra de Barranca I, los antiplásticos esenciales serían los mismos, de origen mineral: cuarzo, mica y feldespato, de una granulometría fina a media y de una distribución más o menos regular. La matriz arcillosa es homogénea pero de momento no se pueden dar más precisiones sin el empleo de microscopía. Finalmente la terminación de superficie es de un alisado expeditivo.

A modo de aproximación al tipo de cocción de la muestra del componente a partir de la observación de las pastas, se siguió una serie de procedimientos de análisis macroscópico en base a lo que García Roselló y Calvo Trias (2006:92) Ilaman: "grupos de trazas macroscópicas de origen térmico, a saber: aspecto cromático, modificaciones térmicas de la forma original de la vasija, aspecto de la pasta cerámica, grietas y fracturas de origen térmico". Dado que resulta difícil identificar deformaciones en la forma original de la vasija por no disponerse de piezas completas, la clasificación inicial se basa en el aspecto cromático siguiendo los 
parámetros del sistema de Munsell (Oyama y Takehara 1970). De esta manera, la coloración predominante en las pastas es marrón grisáceo (7.5YR 4/2), oscilando entre gris pardusco (7.5YR 4/1) y marrón (7.5YR 4/1), con algunos registros de marrón opaco (7.5YR 5/4) y marrón brillante (7.5YR 5/6). Las observaciones indicarían que los contenedores se realizaron mediante una cocción de tipo oxidante, en partes incompleta. Probablemente las imperfecciones y la falta de homogeneidad en el proceso de cocción se deban a su realización en hogueras abiertas, dado que este tipo de cocción presenta mayores variaciones en la temperatura y dificulta el control de la circulación de aire durante el proceso. Elementos como la posición de la pieza y el tipo de combustible empleado también generan variaciones a tener en cuenta. Al no haberse registrado la presencia de áreas de cocción en el sitio, esta es una problemática abierta.

\section{Formas y aspectos tecnológicos}

Dada la fragmentación del registro y la ausencia de colecciones de referencia del área, se partió de identificar inicialmente elementos esenciales: borde, cuerpo y base. Luego se desarrollaron tareas de remontaje y reconstrucción de formas mediante bordes diagnósticos siguiendo a Rice (1987) y Meggers y Evans (1969) y algunos antecedentes en registros de las Sierras de Córdoba, como los trabajos de Pastor (1999) y Dantas y Figueroa (2008).

Estas labores permitieron registrar 32 fragmentos de bordes en la muestra (Figura 2 C, Tabla 2). Se pudieron identificar directos, evertidos e invertidos pero la mayoría resultó inclasificable fehacientemente dado su estado fragmentario. Alrededor del 30\% están decorados. Asimismo, presentan labios directos, convexos y en algunos casos biselados, algunos pocos presentan incisiones en forma de $\mathrm{X}$ o impresiones regulares.

De los 32 bordes apenas 9 permitieron realizar aproximaciones a la forma de los contenedores. Se identificaron 5 formas distribuidas en dos tipos generales, por un lado ollas: esféricas $(\mathrm{N}=1)$, de cuello corto $(\mathrm{N}=2)$ de cuello largo $(\mathrm{N}=3)$; y por otro cántaros: ovaloides rectos $(\mathrm{N}=1)$ y ovaloides evertidos $(\mathrm{N}=2)$.
Esta clasificación permite una aproximación inicial al probable uso de los contenedores de acuerdo a algunas características de performance mecánica y diseño (Hally 1986, Henrickson y McDonald 1983, Rice 1987). Las ollas se asocian generalmente a la cocción de alimentos, dado que al ser globulares facilitan una buena circulación del aire caliente y tienen una mayor resistencia al estrés térmico, su boca restringida evita la pérdida de calor y la evaporación de los contenidos y previene derrames en la manipulación. Contenedores útiles para múltiples propósitos, las ollas son importantes dado que posibilitan el consumo de productos que requieren horas de hervido para ser ingeridos tales como el maíz o la alubia (Medina et al. 2014).

Los cántaros habrían servido más probablemente para el almacenamiento de elementos líquidos y sólidos. Su forma alargada en la que prevalece el alto sobre el diámetro máximo posibilita una eficiente utilización del espacio. Por otra parte no desarrolla las mismas aptitudes que las formas pertenecientes a ollas para la absorción del calor y resistencia al shock térmico, por lo que no habrían sido utilizados para la cocción.

En lo que respecta a los fragmentos de bases identificados (Figura 2 B), estos sugieren que eran biplanas o planoconvexas, sólo se ha podido estimar el diámetro original en un par de ocasiones (en tales casos: $7 \mathrm{~cm}$ y 9,5 cm). En general su espesor es mayor que el del resto de los fragmentos. Entre los atributos de carácter relictual (Rocchietti 1984:81) se identificaron improntas de cestería en dos fragmentos. Para su clasificación, se emplearon la metodología y los conceptos desarrollados por Fabra y Laguens (1999). Asimismo, se tomaron las observaciones metodológicas de López Campeny (2011) y el más reciente trabajo realizado por Figueroa et al. (2011). Se emplearon materiales para obtener un molde en positivo de las improntas en la base y se identificaron los elementos de trama y urdimbre del tejido. La técnica identificada es la de coiled, en la cual la trama se desarrolla a través de la urdimbre tejiéndose alternativamente por arriba y debajo de la misma, lo que conlleva a que esta se vaya torciendo en forma de espiral, hecho que le da su nombre (Fabra y Laguens 1999).

\begin{tabular}{lccccc}
\hline \multirow{2}{*}{ Tipo de Borde } & & \multicolumn{4}{c}{ Tipo de Labio } \\
& Convexo & Recto & Biselado & Inclasificable & Total \\
\hline Evertido & 2 & 0 & 0 & 0 & 2 \\
Recto & 2 & 6 & 1 & 0 & 9 \\
Invertido & 0 & 0 & 1 & 0 & 1 \\
Inclasificable & 1 & 2 & 2 & 15 & 20 \\
Total & 5 & 8 & 4 & 15 & 32 \\
\hline
\end{tabular}

Tabla 2: Tipos de bordes y labios.

Table 2: Types of edges and lips. 


\section{Iconografía}

Finalmente, en lo que respecta a la iconografía, un pequeño porcentaje de los tiestos está decorado, 28 tiestos (casi un 6\% de la muestra total) de los cuales la mitad corresponden a fragmentos de bordes. Se siguió una vía inicial para su estudio en base a los parámetros clásicos establecidos por Shepard (1985:261-267): campo decorativo, composición y estructura, elementos o motivos. Procurando identificar las técnicas aplicadas. En función de esto, se pudo consignar que los elementos o motivos de la decoración en los tiestos del Componente I del sitio son esencialmente dos: líneas (rectas o zigzagueantes) y puntos, trazados mediante las técnicas de incisión e impresión respectivamente, en general de un trazo fino y superficial bien definido, realizado con un objeto punzante. Respecto a su composición y estructura se destacan aquellos fragmentos en los que se esbozan figuras geométricas -rectangulares o triangulares-, con líneas incisas encerrando las impresiones o puntos al interior. Finalmente, un buen número de los bordes identificados presenta decoración, lo que da una idea de uno de los campos decorativos que han sido empleados frecuentemente.

\section{Consideraciones finales}

A modo de síntesis los estudios realizados hasta el momento permiten decir que la cerámica del Componente I del sitio Barranca manifiesta cierta uniformidad en los aspectos macroscópicos, con materias primas de disponibilidad local, probablemente arenas y arcillas de las márgenes e inmediaciones del río Piedra Blanca. Los antiplásticos seleccionados en general son los mismosmica, cuarzo y feldespato- con homogeneidad relativa en granulometría, distribución y abundancia. De acuerdo a las variaciones cromáticas en pasta la cocción habría sido realizada en hogueras abiertas, posiblemente mediante combustibles vegetales-aunque para corroborar esto se requiere de otros análisis complementarios-.

Por otra parte, la variedad de formas identificadas sugieren un grado mínimo de especialización funcional de los contenedores, en los que la decoración se presenta en baja proporción, con motivos similares entre sí, con presencia de improntas cesteras de tipo coiled en las bases. Estos aspectos sumados a la presencia de torteros y al fragmento de estatuilla permiten establecer correlaciones entre el conjunto tecnológico de este componente del Barranca con los registros de otros sitios del Holoceno Tardío, en las Sierras Centrales de Córdoba.

Finalmente, considerando la necesidad de avanzar en estudios microscópicos, los indicios actuales permiten afirmar que la recurrencia y la variabilidad de los atributos identificados en la muestra -en los procedimientos de manufactura, en la composición de las pastas, morfología y decoración- estarían dando cuenta de una alfarería de elaboración expeditiva -especialmente en su terminación- a partir de materias primas disponibles en el entorno ambiental inmediato, y posiblemente orientada al aprovechamiento de los recursos silvestres del bosque del espinal.

Río Cuarto, 14 de Mayo de 2016.

\section{Referencias bibliográficas}

Austral, A. \& A. Rocchietti. 2004. Al sur del río Cuarto: Síntesis de la Arqueología Regional. En: Bechis, M. (Comp.) Terceras Jornadas de Arqueología Histórica y de Contacto del Centro Oeste de la Argentina Seminario de Etnohistoria. Cuartas Jornadas de Arqueología y Etnohistoria del Centro Oeste del País. Universidad Nacional de Río Cuarto. Río Cuarto. Pp. 97- 114.

Cremonte, M. B. \& M. F. Bugliani. 2006-2009. Pasta, forma e iconografía. Estrategias para el estudio de la cerámica arqueológica. Xama 19-23:239-262.

Dantas, M. \& G. Figueroa. 2008. Análisis tecnológico y funcional del registro cerámico del Valle de Salsacate y Pampas de altura adyacentes (Provincia de Córdoba, República Argentina). British Archaeological Reports, International Series S1869. Oxford.

Fabra, M. \& A. Laguens. 1999. Análisis tecnológico de improntas de cestería en fragmentos cerámicos de Córdoba, Argentina. En Actas del XII Congreso Nacional de Arqueología Argentina. La Plata. Tomo II. 25-34.

Figueroa, G.; E. Pautassi \& M. Dantas. 2011. Técnicas cesteras y cerámica arqueológica de las Sierras Centrales de Córdoba, República Argentina. ArqueoWeb. Revista sobre Arqueología en Internet 13:15-31.

García Roselló, J. \& M. Calvo Trias. 2006. Análisis de las evidencias macroscópicas de cocción en la cerámica prehistórica: una propuesta para su estudio. Mayurqa (31): 83-112.

Hally, D. J. 1986. The identification of vessel function: A case study from Northwest Georgia. American Antiquity 51 (2): 267:295

Henrickson, E. F. \& M. M. McDonald. 1983. Ceramic Form and Function: an ethnographic search and archaeological application. American Antiquity 51 (2): 267-295.

Lopez Campeny, S. 2011. La impresión es lo que cuenta... análisis de improntas textiles. Casos arqueológicos para Santiago del Estero. Relaciones de la Sociedad Argentina de Antropología XXXVI: 221-247.

Medina, M.; S. Pastor \& E. Berberián. 2014. Es gente fazil de moverse de una parte a otra. Diversidad en las 
estrategias de subsistencia y movilidad prehispánicas tardías (Sierras de Córdoba, Argentina). Complutum 25 (1): 73.88 .

Meggers, B. \& C. Evans. 1969. Como interpretar el lenguaje de los tiestos. Smithsonian Institution.

Oyama, M. \& H. Takehara. 1970. Revised standard soil color charts. Revised Edition Japan Colour Research Institute. Tokyo.

Pastor, S. 1999. Forma y función de las vasijas de San Roque (Dpto. Punilla, Pcia de Córdoba). En Actas del XII Congreso Nacional de Arqueología Argentina. La Plata. Tomo III. Pp. 511-516.

Pastor, S. \& M. Medina. 2013. Prácticas resistentes, elusión y reproducción social en un contexto histórico adverso. Una mirada a los indígenas de Córdoba (Argentina) en tiempos Coloniales Tempranos. Memoria Americana. Cuadernos de Etnohistoria 21 (1): 66-92.

Rice, P. M. 1987. Pottery Analysis: a sourcebook. University of Chicago Press. Chicago.

Rocchietti, A. 1984. Ordenes de la clasificación arqueológica: sus fundamentos ideográficos y nomotéticos. Revista UNRC (1): 75-112.

Rocchietti, A. y\&F. Ribero 2015a. "Fechados radiocarbónicos y distirbuciones arqueológicas en localidades de la Sierra de Comechingones (provincial de Córdoba)". En Pifferetti, A. \& I. Dosztal (Comps.) Arqueometría Argentina. Metodologías científicas aplicadas al studio de los bienes culturales: datación, caracterización, prospección y conservación. Aspha. Buenos Aires.

Rocchietti, A. \& F. Ribero 2015b. Sitio Barranca I: Sistemática estratigráfica de depósito Ceramolítico en el piedemonte de la Sierra de Comechingones, provincia de Córdoba. Trabajo presentado en el VI Congreso Nacional de Arqueometría. Río Cuarto, Diciembre de 2015. Inédito.

Rocchietti, A., M. Tamagnini, E. Olmedo, G. Perez Zavala, F. Ribero, A. Ponzio, L. Alaniz, D. Reinoso, A. Cavallin, P. Altamirano \& A. Ponce. 2013. La formación del territorio surcordobés a través de su potencial arqueológico. Plan director Achiras histórica. Revista cultura en red. I (I): 101-131.

Shepard, A. O. 1985. Ceramics for the archaeologist. Carnegie Institute of Washington. 